\title{
Measuring patient-reported outcomes in psychosis: conceptual and methodological review
}

Ulrich Reininghaus and Stefan Priebe

\section{Background}

There are calls to use patient-reported outcomes (PROS) routinely across mental health services. However, the use of PROs in patients with psychosis has been questioned.

\section{Aims}

To examine the concepts and measures of four widely used PROs: treatment satisfaction, subjective quality of life, needs for care and the quality of the therapeutic relationship.

\section{Method}

We conducted a literature search of academic databases on concepts, characteristics and psychometric properties of the four PROs in patients with psychosis.

\section{Results}

Although numerous concepts and measures have been published, evidence on the methodological quality of existing PROs is limited. Measures designed to assess distinct PROs showed a considerable conceptual, operational and empirical overlap, and some of them also included specific aspects. The impact of symptoms and cognitive deficits appears unlikely to be of clinical significance.

\section{Conclusions}

The popularity of PROs has not been matched with progress in their conceptualisation and measurement. Based on current evidence, some recommendations can be made. Distinct and short measures with clinical relevance and sufficient psychometric properties should be preferred. Future research should optimise the validity and measurement precision of PROs, while reducing assessment burden.

\section{Declaration of interest}

None.
Since the 1960s, patient-reported outcomes (PROs) have become increasingly popular in the care of patients with psychosis. There is no universally accepted terminology and definition of such outcomes. In the literature the terms 'PROs', 'patient-reported outcome measures' (PROMs), 'patient-based outcomes', 'patientdriven outcomes', 'self-rated outcomes' and 'subjective evaluation criteria' have been used interchangeably. ${ }^{1-3}$ In recent years the term 'PRO' appears to be most widely used. ${ }^{2}$ The US Food and Drug Administration (FDA) defined PROs as:

'any report of the status of a patient's health condition that comes directly from the patient, without interpretation of the patient's response by a clinician or anyone else' (p. 2). ${ }^{4}$

Treatment satisfaction, subjective quality of life (SQoL), needs and the quality of the therapeutic relationship can be considered as four historically rooted, commonly used and important PRO concepts in the care of patients with psychosis. ${ }^{2,5,6}$ Whereas the list of PROs has increased steadily, their popularity has gained momentum over the past decade, partly through their intuitive appeal for stakeholder groups. ${ }^{3-5}$ In the UK a recent National Health Service (NHS) White Paper announced plans for new outcome assessments, in which PROs were to be used to measure the effectiveness of services. ${ }^{3}$ Using PROs in the monitoring of outcomes of individual patients and services can also feed into the patient-clinician communication, reflective practice, quality management and service development. ${ }^{78}$ However, the selection of appropriate concepts and measures often remains difficult. Further, some authors have questioned the use of PROs in patients with psychosis owing to conceptual and methodological shortcomings, ${ }^{9}$ with some proposing to discard them entirely. ${ }^{10}$ Against this background, this review aimed to examine the concepts and measures of four widely used PROs - treatment satisfaction, SQoL, needs for care and the quality of the therapeutic relationship - in the evaluation of care of patients with psychosis.

\section{Method}

A review of the conceptual and methodological literature on the four PROs in the care of patients with psychosis was conducted. We searched the literature systematically and also followed the recommendations for conceptual and methodological reviews to search widely in disparate sources and allow for overlap in the various stages (literature search, analysis and writing). ${ }^{11,12}$

\section{Search strategy and selection criteria}

A search of the academic databases EMBASE, Medline and PsycINFO was performed to identify papers that, first, reported the characteristics and psychometric properties of PRO measures to assess treatment satisfaction, SQoL, needs for care and the therapeutic relationship in the care of patients with psychosis, and second, provided definitions of concepts intended to be assessed by at least one of the identified measures. The term 'PRO' was used in accordance with the FDA definition given earlier. The literature search combined three groups of keywords in each database:

(a) schizophr ${ }^{\star}$, psychosis OR psychoses;

(b) quality of life, subjective quality of life, treatment satisfaction, patient satisfaction, need ${ }^{*}$, therapeutic relationship, therapeutic alliance, helping alliance OR working alliance;

(c) psychometric ${ }^{\star}$, validity, reliability OR responsiveness.

Titles and abstracts were screened and papers retrieved to assess their relevance. Reference lists of relevant papers were inspected for additional papers. References that cited previously identified papers were searched using the 'cited by' option in the electronic database Web of Science. In addition to the search 
of academic databases, informal networks were used to identify papers.

\section{Data extraction and synthesis}

As the conceptual and methodological literature on PROs in the evaluation of treatments for psychosis is vast and disparate, a quantitative synthesis appeared neither appropriate nor feasible. The findings are presented descriptively. Although PRO measures can be distinguished according to various characteristics, we focused on the following ones: concept purported to be measured, number and content of domains, estimated completion time, response options and type (generic, condition- or disease-specific, treatment-specific and utility measures). ${ }^{4,13}$ Numerous psychometric properties for evaluating PROs have been proposed in the literature. ${ }^{14}$ We distinguished between reliability (i.e. internal consistency, reliability, scale information), validity (i.e. content validity, including face validity, and construct validity, including structural, convergent, discriminant, cross-cultural, concurrent and predictive validity) and responsiveness. ${ }^{14}$ Given the lack of consensus on how these psychometric properties are best evaluated and findings synthesised, ${ }^{4,13}$ we used a simple, dichotomous rating of whether or not a psychometric property had been examined for a given instrument.

\section{Results}

The results of the search strategy are summarised in Fig. 1. The search initially yielded a total of 2181 items ( 813 duplicates). Titles

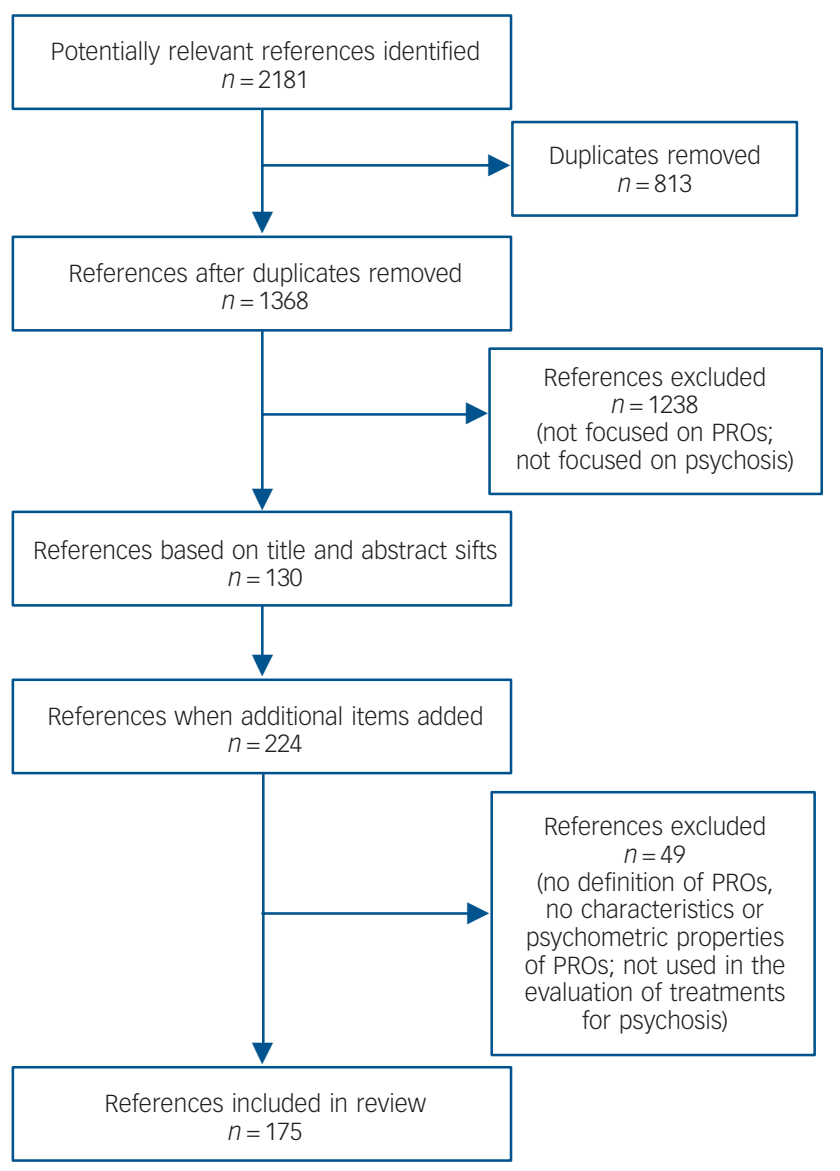

Fig. 1 Results of database search. PRO, patient-reported outcome. and abstracts were screened for 1368 references. Based on title and abstract sifts, 1238 references were excluded because they did not focus on the four PROs or psychosis. The number of potentially relevant references increased from 130 to 224 when additional items were added. Of these, 49 references were excluded for different reasons. Hence, from the 2181 initially identified references, only 175 were included in our review.

\section{Concepts and definitions}

Definitions of concepts to be assessed by the identified PRO measures of treatment satisfaction, SQoL, needs for care and the therapeutic relationship are summarised in online Table DS1. Measures of treatment satisfaction for which a definition of the concept to be measured was provided all purported to assess the multidimensional satisfaction concept of a personal evaluation of healthcare services and providers as proposed by Ware et al and Ruggeri et al. ${ }^{15,16}$ The identified SQoL measures were intended to assess a range of concepts (Table DS1). The only measure of needs that provided a definition of the concept to be measured, the Camberwell Assessment of Need (CAN), ${ }^{17}$ purported to assess a supply and perceived need concept. Pantheoretical, ${ }^{18}$ Rogerian, ${ }^{19}$ systemic $^{20}$ and psychoanalytic $^{21,22}$ concepts were intended to be assessed by the identified measures of the therapeutic relationship. For each of the four PROs, no single universally accepted definition could be identified. Nevertheless, there were attempts to identify a common conceptual basis. Lauer noted that:

'There is agreement that quality of life is a multi-dimensional phenomenon and construct, aiming at a holistic or global perspective of individuals in their biopsychosocial nature' (p. 19). ${ }^{23}$

Similarly, Ware et al emphasised that treatment satisfaction is most widely measured as a multidomain concept. ${ }^{15}$ However, this may imply a risk of providing non-specific or overinclusive definitions. Several PRO concepts other than the one to be measured may meet very broad definitions; for example, Stevens \& Gabbay defined needs as 'the ability to benefit in some way from health care' (p. 21). ${ }^{24}$ Others have found a lack of clarity of the precise nature of some PRO concepts:

'In psychiatry, there is as yet no clearly defined concept of the therapeutic alliance' (Catty: p. 265). ${ }^{25}$

A tendency was found to use terms from different theoretical backgrounds and traditions with at least slightly different connotations synonymously. For example, the term 'therapeutic relationship' has been used interchangeably with the terms 'therapeutic alliance', 'helping alliance' or 'working alliance', each of which has emerged from different lines of research. ${ }^{25}$ Similarly, 'treatment satisfaction' has been used synonymously with 'patient satisfaction', 'service satisfaction' and 'satisfaction with care', to name a few. ${ }^{15,16}$ This may lead to a lack of clarity as to precisely which conceptualisation of PROs is being referred to. ${ }^{26}$ Several definitions of PRO concepts were found to overlap with others (Table DS1). However, some definitions of PRO concepts did not, and contained specific elements: this applied to definitions of SQoL, ${ }^{27,28}$ needs for care, ${ }^{29}$ and the therapeutic relationship. ${ }^{18,21,22}$ Overall, definitions of PRO concepts were found to vary in the extent to which they included overlapping and specific aspects.

\section{Characteristics of PRO measures}

Findings on characteristics and psychometric properties of PRO measures to assess treatment satisfaction, SQoL, needs for care and the therapeutic relationship are summarised respectively in online Tables DS2-DS5. For several measures the concept that the measure was intended to assess was not provided. Most 
measures were generic in nature and used Likert scales. Short versions have been developed for several measures, based on conceptual and practical rather than empirical considerations. A number of measures were found to be long and time-consuming to administer: several had more than 30 items and a completion time greater than $20 \mathrm{~min}$.

Several PRO measures were intended to assess multidomain concepts, with items being grouped within domains, and domains within more general PRO concepts. An overlap in the content of domains was observed across measures that were intended to assess different PROs. Specifically, the domains of measures to assess SQoL are similar and in part even identical to domains included in measures of needs. This applies likewise to measures of treatment satisfaction and the therapeutic relationship. The content of domains of treatment satisfaction and needs for care measures, and the content of treatment satisfaction and SQoL measures, show substantial overlap (Tables DS2-DS5).

\section{Psychometric properties of PRO measures}

The evaluation of the reviewed measures often included only limited information on psychometric properties in patients with psychosis (Tables DS2-DS5). The methods used to assess structural validity were largely not appropriate for ordinal data, as required for the predominantly used Likert scales. ${ }^{30}$ Only for two measures, the Quality of Life Interview (QoLI) and EuroQoL-5D, ${ }^{31-34}$ was there evidence on structural validity based on confirmatory factor analysis for ordinal data or item response modelling. ${ }^{30,35,36}$ For most measures there was no evidence on their measurement precision throughout the full range of scores; only for the QoLI was this psychometric property examined. ${ }^{36}$ For some measures no evidence on their internal consistency, test-retest reliability and scale information as well as content, structural, discriminant, convergent, concurrent, predictive or cross-cultural validity was found in the included studies.

\section{Empirical overlap of PRO measures}

Only a few studies assessed more than one outcome at a time. They consistently suggest low discriminant validity due to an empirical overlap of measures designed to assess different outcomes. The outcomes were substantially correlated, ${ }^{37-41}$ and a single general factor explained more than half of the variance in SQoL, needs for care and treatment satisfaction scores., ${ }^{2,42,43}$ The general factor has been interpreted as a general appraisal tendency of patients for positive or negative ratings across measures designed to assess different PRO concepts. ${ }^{42}$ However, this general appraisal tendency left about half of the variance unexplained, which is potentially concept-specific. A recent study suggested a bifactor model which confirms the importance of a general appraisal tendency, but also shows the relevance of concept-specific aspects. The latter provide distinct information that is independent from both the general appraisal tendency and other concepts. ${ }^{44}$

\section{Association with psychiatric symptoms and cognitive deficits}

There was also evidence from several studies that less favourable SQoL is related to higher levels of psychopathologic disorder including positive, negative and depressive symptoms. ${ }^{45-56}$ For the association of psychiatric symptoms and treatment satisfaction, Katsakou \& Priebe reported an inverse relationship between psychiatric symptoms and level of treatment satisfaction, ${ }^{57}$ which is in line with other studies. ${ }^{58}$ There are also a number of studies suggesting that patients with more severe psychotic symptoms have more unmet and total needs for care. $^{59-61}$ However, a more recent pooled analysis of individual patient-level data obtained from 16 studies found that symptom levels were less strongly associated with SQoL in schizophrenia than in other mental disorders. ${ }^{62}$ A pooled analysis of associations between changes of symptoms and SQoL ratings over time identified an explained variance of only $5.5 \% .{ }^{63}$

With respect to cognitive deficits, evidence on associations with PROs remains inconsistent. Fujii et al found that better cognitive performance was associated with lower SQoL ratings in a prospective study of patients with severe and enduring psychosis, ${ }^{64}$ which is consistent with other studies. ${ }^{50,65-70}$ However, Galletly et al, Ritsner and Sota found the opposite. ${ }^{71-73}$ Deficits in executive functioning, attention, memory and motor skills were associated with lower SQoL. One recent study on bias of PRO ratings by psychiatric symptoms and cognitive deficits at the item level identified no effect of cognitive deficits on the responses to single items and no effect of symptoms on the responses of only two single items. The study concluded that the magnitude of any response bias through symptoms or cognitive deficits, if present, is small and unlikely to be of clinical significance. $^{74}$

\section{Discussion}

Our review examining concepts and measures of four established PROs in the evaluation of treatments for psychosis generated at least three important findings. First, despite the increasing popularity of PROs with numerous concepts and measures, evidence of their methodological quality remains limited. Second, there is a considerable conceptual, operational and empirical overlap across measures designed to assess different PROs, although some concepts and measures also included aspects specific to individual PROs. Last, the influence of (or bias by) cognitive deficits and psychiatric symptoms appears limited and unlikely to be of clinical significance.

\section{Limitations}

The review has several limitations. The findings may be biased, as important references on concepts, characteristics and psychometric properties of PRO measures may have been missed. Concepts that might be relevant for one of the four PROs, but were not captured in an existing measure, were not included. The review was selective in examining concepts and measures of only four PROs and only a limited number of psychometric properties. Although Mokkink et al achieved a degree of consensus on the terminology and definitions of psychometric properties and provided guidance on data synthesis for reviews of the methodological quality of studies investigating psychometric properties of PROs, ${ }^{14}$ there is no consensus on how to synthesise findings on psychometric properties per se. We classified PROs according to whether or not they assessed specific psychometric properties. Given the absence of a consensus, this did not include ratings of the extent to which these psychometric properties were met. Finally, given the nature of conceptual and methodological reviews, ${ }^{11,14}$ there may have been a subjective bias of the authors in the analysis and interpretation of the literature.

\section{Methodological quality of PROs}

Over the past decades numerous concepts and measures of PROs have emerged. ${ }^{75-77}$ In contrast, our review found only limited evidence of their methodological quality. Several measures were not linked to specific concepts. A number of measures were long 
and time-consuming to administer. This may imply undue assessment burden on patients with psychosis as well as increased assessment costs. For most measures there was no evidence on their measurement precision throughout the full range of scores, as has been established by a few studies for observer-rated outcome measures in mental health, ${ }^{78}$ and, on a larger scale, for PROs in other medical disorders. ${ }^{79}$ The methods used to assess structural validity were largely not appropriate for ordinal data. ${ }^{80}$ Only a few of the reviewed studies conducted analyses based on confirmatory factor analysis for ordinal data or item response modelling. ${ }^{80,81}$ There are several implications of treating ordinal data as continuous, including attenuated relationships among PRO items in the presence of floor or ceiling effects, presence of pseudofactors and incorrect parameter estimates. ${ }^{82}$ These may challenge findings on the structural validity of PRO measures. In other words, measures using Likert scales, which have not been examined with psychometric methods appropriate for ordinal data, may be impaired in their ability to summarise patients' item responses into scores that adequately reflect their dimensional structure. This is, however, central for the use of PROs in the evaluation of care, as such scores provide the basis on which value is assigned to treatments.

\section{Conceptual, operational and empirical overlap}

The conceptual, operational and empirical overlap of PROs has several implications for the validity of existing PRO measures. Campbell \& Fiske, in their seminal work on discriminant and convergent validity, stated:

'One cannot define without implying distinctions, and the verification of these distinctions is an important part of the validational process' (p. 84). ${ }^{83}$

The verification of distinctions appears to be a part of the validational process that has been neglected by most of the research into PROs. New concepts were often proposed without assessing whether they were sufficiently distinct from existing concepts to warrant them being measured separately. This review suggests that an insufficient distinction between PROs at the conceptual level has led to a considerable overlap in the content of specific domains. This implies that, both at a conceptual and operational level, the requirements for establishing discriminant validity were not sufficiently considered when developing PROs. Empirically this may limit the ability of established measures to capture variance specific to the given concept. Indeed, this points towards substantial empirical overlap across measures. Although such overlap may reflect real associations between different PROs (e.g. one PRO influencing another), it still impairs the ability of each PRO measure to capture distinct information and, in psychometric terms, their discriminant validity. ${ }^{83}$ However, some concepts and operationalisations included aspects that were specific to one or more PROs. Recent evidence suggests that PROs may reflect both a general appraisal tendency that uniformly influences all PRO ratings in a positive or negative direction and components that are specific for each PRO. The specific information is independent of the general appraisal tendency. Maximising the specific information may be a challenge for future scale improvements.

\section{Influence of cognitive deficits and psychiatric symptoms}

In contrast to the concerns of some authors that the validity of existing PRO measures might be impaired owing to the influence of psychiatric symptoms and cognitive deficits, ${ }^{9,10}$ findings from our review suggested that the influence of (or bias by) cognitive deficits and psychiatric symptoms is very limited. The identified associations of PROs with symptoms and deficits do not compromise their validity as independent outcome criteria. However, all the evidence was taken from patients who consented to participate in research and were seen as capable of providing reasonable responses. Patients with high symptom levels may have been excluded from such studies, by clinicians or researchers. There is no evidence of a possible threshold of general or specific symptoms above which PROs might yield less reliable results.

\section{Routine use of PROs}

The conceptualisation and measurement of PROs in patients with psychosis are of practical relevance. These measures have an intuitive appeal for various stakeholder groups and there are calls to use them routinely across mental health services. ${ }^{3}$ Even though evidence on the methodological quality of PROs is limited overall, there are at least five recommendations that can be made about the routine use of PROs in the evaluation of treatments for psychosis.

(a) It should be carefully considered which PRO is relevant to the aim and approach of the given service, and what the implications of its results would be for service delivery and development.

(b) The use of several PRO measures should be avoided unless they address clearly distinct domains.

(c) Measures with evidence of good psychometric properties should be preferred; the evidence on psychometric properties is limited for most measures. Overall, measures using satisfaction-based concepts (e.g. assessing satisfaction with life domains or with treatment) have been more rigorously studied than others.

(d) In the absence of evidence showing that longer measures have superior properties, shorter measures should be prioritised to minimise the burden and costs of measurement. However, longer measures tend to be more reliable, and there can be a trade-off between brevity and psychometric quality.

(e) The influence of symptoms and cognitive deficits is unlikely to affect findings in small samples (although even a small explained variance may be relevant for research in large samples).

\section{Future research}

Despite the popularity of PROs for measuring the quality of routine mental healthcare, there are a number of conceptual and methodological shortcomings. Although according to our main findings this includes considerable conceptual, operational and empirical overlap across measures designed to assess different PROs, the influence of cognitive deficits and psychiatric symptoms appears limited. There is a need for more rigorous research to identify short measures that assess distinct PROs independent from overlap with highest possible precision. New methods such as item response modelling, item banking and computerised adaptive testing may help move this forward. ${ }^{74,79}$ Although such methods have been infrequently used in psychiatric studies, ${ }^{78}$ they have led to progress in measuring PROs in other medical conditions. A prominent example is the Patient-Reported Outcomes Measurement Information System (PROMIS). ${ }^{79}$ Computerised adaptive testing iteratively selects the item providing the highest precision for a given patient until a desired level of precision is achieved. This minimises the number of items each patient has to complete, ${ }^{84}$ and can be implemented on handheld electronic 
devices. Ideally, conceptual and methodological work should be linked in future research to advance the measurement of PROs in patients with psychosis, so that concepts can both inform research and be refined on the basis of empirical data.

Ulrich Reininghaus, PhD, King's College London, Institute of Psychiatry; Stefan Priebe, FRCPsych, Queen Mary University of London, Unit for Social and Community Psychiatry, Barts and the London School of Medicine, UK

Correspondence: Dr Ulrich Reininghaus, Section of Social Psychiatry, PO Box 33, Health Service and Population Research Department, Institute of Psychiatry, De Crespigny Park, London SE5 8AF, UK. Email: ulrich.reininghaus@kcl.ac.uk

First received 24 Jan 2012, final revision 18 Mar 2012, accepted 3 Apr 2012

\section{Funding}

This work was supported by a research training fellowship funded by the UK Nationa Institute of Health Research to U.R. The report is independent research and the views expressed in this publication are those of the authors and not necessarily those of the NHS, the National Institute for Health Resaerch or the Department of Health.

\section{References}

1 Priebe S, Gruyters T, Heinze M, Hoffmann C, Jakel A. Subjective evaluation criteria in psychiatric care - methods of assessment for research and general practice [in German]. Psych Prax 1995; 22: 140-4.

2 Hansson L, Bjorkman T, Priebe S. Are important patient-rated outcomes in community mental health care explained by only one factor? Acta Psychiatr Scand 2007; 116: 113-8.

3 Department of Health. Equity and Excellence: Liberating the NHS. Department of Health, 2010.

4 US Food \& Drug Administration. Guidance for Industry. Patient-reported Outcome Measures: Use in Medical Product Development to Support Labeling Claim. FDA, 2009

5 McCabe R, Saidi M, Priebe S. Patient-reported outcomes in schizophrenia. $\mathrm{Br}$ J Psychiatry 2007; 191 (suppl 50): s21-8.

6 Kilian R, Angermeyer M. Quality of life in psychiatry as an ethical duty: from the clinical to the societal perspective. Psychopathology 1999; 32: 127-34.

7 Slade M, McCrone P, Kuipers E, Leese M, Cahill S, Parabiaghi A, et al. Use of standardised outcome measures in adult mental health services. Randomised controlled trial. Br J Psychiatry 2006; 189: 330-6.

8 Priebe S, McCabe R, Bullenkamp J, Hansson L, Lauber C, Martinez-Leal R, et al. Structured patient-clinician communication and 1-year outcome in community mental healthcare. Cluster randomised controlled trial. Br J Psychiatry 2007; 191: 420-6.

9 Atkinson $\mathrm{M}$, Zibin S, Chuang $\mathrm{H}$. Characterizing quality of life among patients with chronic mental illness: a critical examination of the self-report methodology. Am J Psychiatry 1997; 154: 99-105.

10 Epstein A, Hall J, Tognetti J, Son L, Conant L. Using proxies to evaluate quality of life: can they provide valid information about patients' health status and satisfaction with medical care? Med Care 1989; 27 (suppl 3): s91-8.

11 Lilford R, Richardson A, Stevens A, Fitzpatrick R, Edwards S, Rock F, et al. Issues in methodological research: perspectives from researchers and commissioners. Health Technol Assess 2001; 5: 1-57.

12 Morgan C, Burns T, Fitzpatrick R, Pinfold V, Priebe S. Social exclusion and mental health. Conceptual and methodological review. Br J Psychiatry 2007; 191: 477-83.

13 Fitzpatrick R, Davey C, Buxton M, Jones D. Evaluating patient-based outcome measures for use in clinical trials. Health Technol Assess 1998; 2.

14 Mokkink L, Terwee C, Patrick D, Alonso J, Stratford P, Knol D, et al. The COSMIN study reached international consensus on taxonomy, terminology, and definitions of measurement properties for health-related patientreported outcomes. J Clin Epidemiol 2010; 63: 737-45.

15 Ware J, Snyder M, Wright W, Davies A. Defining and measuring patient satisfaction with medical care. Eval Progr Plan 1983; 6: 247-63.

16 Ruggeri M, Dall'Agnola R, Agostini C, Bisoffi G. Acceptability, sensitivity and content validity of the VECS and VSSS in measuring expectations and satisfaction in psychiatric patients and their relatives. Soc Psychiatry Psychiatr Epidemiol 1994; 29: 265-76.

17 Phelan M, Slade M, Thornicroft G, Dunn G, Holloway F, Wykes T, et al. The Camberwell Assessment of Need: the validity and reliability of an instrument to assess the needs of people with severe mental illness. Br J Psychiatry 1995; 167: 589-95.

18 Bordin E. The generalizability of the psychoanalytic concept of the working alliance. Psychotherapy 1976; 16: 252-60.

19 Priebe S, Gruyters T. The role of the helping alliance in psychiatric community care. A prospective study. J Nerv Ment Dis 1993; 181: 552-7.

20 Pinsof W, Catherall D. The integrative psychotherapy alliance: family, couple and individual therapy scales. J Marital Fam Therapy 1986; 12: 137-51.

21 Freud S. On beginning the treatment. Reprinted (1953-1974) in the Standard Edition of the Complete Psychological Works of Sigmund Freud (trans \& ed J Strachey), vol 12. Hogarth Press.

22 Sterba R. The fate of the ego in analytic therapy. Int J Psychoanalysis 1934; 115: $117-26$.

23 Lauer G. Concepts of quality of life in mental health care. In Quality of Life in Mental Health Care (eds S Priebe, J Oliver, W Kaiser): 19-34. Wrightson, 1999.

24 Stevens A, Gabbay J. Needs assessment needs assessment. Health Trends 1991; 23: 20-3.

25 Catty J. 'The vehicle of success': theoretical and empirical perspectives on the therapeutic alliance in psychotherapy and psychiatry. Psychol Psychother 2004; 77: 255-72.

26 Catty J, Winfield $\mathrm{H}$, Clement S. The therapeutic relationship in secondary mental health care: a conceptual review of measures. Acta Psychiatr Scand 2007; 116: 238-52.

27 Zautra A, Goodhard D. Quality of life indicators: a review of the literature. Commun Ment Health Rev 1979; 4: 3-10.

28 Clare A, Corney R, Cairns V. Social adjustment: the design and use of an instrument for social work and social work research. Br J Soc Work 1984; 14 323-36.

29 Department of Health Social Services Inspectorate. Care Management and Assessment: Summary of Practice Guidance. HMSO, 1991.

30 Gibbons R, Bock D, Hedeker D, Weiss D, Segawa E, Bhaumik D, et al. Full-information bifactor analysis for graded response data. Appl Psychol Meas 2007; 31: 4-19.

31 Lehman A. A quality of life interview for the chronically mentally ill. Eval Progr Plan 1988; 11: 51-62.

32 Lehman A, Ward N, Linn L. Chronic mental patients: the quality of life issue Am J Psychiatry 1982; 139: 1271-6.

33 Brooks R. EuroQol: the current state of play. Health Policy 1996; 37: 53-72.

34 EuroQol Group. EuroQol - a new facility for the measurement of healthrelated quality of life. Health Policy 1990; 16: 199-206.

35 Prieto L, Novick D, Sacristan J, Edgell G. A Rasch model analysis to test the cross-cultural validity of the EuroQoL-5D in the Schizophrenia Outpatient Health Outcomes study. Acta Psychiatr Scand 2003; 107: 24-9.

36 Uttaro T, Lehman A. Graded response modeling of the Quality of Life Interview. Eval Progr Plan 1999; 22: 41-52.

37 De Weert-van Oene G, Havenaar J, Schrijvers A. Self-assessment of need for help in patients undergoing psychiatric treatment. Psychiatry Res 2009; 167: 221-30.

38 Lasalvia A, Bonetto C, Tansella M, Stefani B, Ruggeri M. Does staff-patien agreement on needs for care predict a better mental health outcome? A 4-year follow-up in a community service. Psychol Med 2008; 38: 123-33.

39 McCabe R, Roder-Wanner U, Hoffmann K, Priebe S. Therapeutic relationships and quality of life: association of two subjective constructs in schizophrenia patients. Int J Soc Psychiatry 1999; 45: 276-83.

40 Priebe S, Mccabe R. The therapeutic relationship in psychiatric settings. Acta Psychiatr Scand Suppl 2006; 429: 69-72.

41 Slade M, Leese M, Cahill S, Thornicroft G, Kuipers E. Patient-rated mental health needs and quality of life improvement. Br J Psychiatry 2005; 187: 256-61.

42 Priebe S, Kaiser W, Huxley $\mathrm{P}$, Roder-Wanner U, Rudolf H. Do different subjective evaluation criteria reflect distinct constructs? J Nerv Ment Dis 1998; 186: 385-92.

43 Fakhoury W, Kaiser W, Roeder-Wanner U, Priebe S. Subjective evaluation: is there more than one criterion? Schizophr Bull 2002; 28: 319-27.

44 Reininghaus $U$, McCabe R, Burns T, Croudace T, Priebe S. Measuring patients' views: a bi-factor model of distinct patient-reported outcomes in psychosis. Psychol Med 2011; 41: 277-89.

45 Norholm V, Bech P. Quality of life in schizophrenic patients: association with depressive symptoms. Nord J Psychiatry 2006; 60: 32-7.

46 Oersel S, Akdemir A, Dag I. The sensitivity of quality-of-life scale WHOQOL-100 to psychopathological measures in schizophrenia. Compr Psychiatry 2004; 45: 57-61. 
47 Wilkinson G, Hesdon B, Wild D, Cookson R, Farina C, Sharma V, et al. Self-report quality of life measure for people with schizophrenia: the SQLS. Br J Psychiatry 2000; 177: 42-6.

48 Endicott J, Nee J, Harrison W, Blumenthal R. Quality of Life Enjoyment and Satisfaction Questionnaire: a new measure. Psychopharmacol Bull 1993; 29 : 321-6.

49 Ritsner M, Kurs R, Ratner Y, Gibel A. Condensed version of the Quality of Life Scale for schizophrenia for use in outcome studies. Psychiatry Res 2005; 135: $65-75$.

50 Corrigan $\mathrm{P}$, Buican $\mathrm{B}$. The construct validity of subjective quality of life for the severely mentally ill. J Nerv Ment Dis 1995; 183: 281-5.

51 Eack S, Newhill C. Psychiatric symptoms and quality of life in schizophrenia. Schizophr Bull 2007; 33: 1225-37.

52 Mechanic D, McAlpine D, Rosenfield S, Davis D. Effects of illness attribution and depression on the quality of life among persons with serious mental illness. Soc Sci Med 1994; 39: 155-64.

53 Pukrop R, Schlaak V, Moller-Leimkuhler A, Albus M, Czernik A, Klosterkotte $\mathrm{J}$, et al. Reliability and validity of quality of life assessed by the Short-Form 36 and the Modular System for Quality of Life in patients with schizophrenia and patients with depression. Psychiatry Res 2003; 119: 63-79.

54 Price M, Hill C, Williams V, Morlock R, Leeuwenkamp O, Patterson T. Measures of functional status and quality-of-life in schizophrenia. Curr Psychiatry Rev 2008; 4: 28-38.

55 Sim K, Mahendran R, Siris S, Heckers S, Chong S. Subjective quality of life in first episode schizophrenia spectrum disorders with comorbid depression. Psychiatry Res 2004; 129: 141-7.

56 Vatne S, Bjorkly S. Empirical evidence for using subjective quality of life as an outcome variable in clinical studies: a meta-analysis of correlates and predictors in persons with a major mental disorder living in the community Clin Psychology Rev 2008; 28: 869-89.

57 Katsakou C, Priebe S. Outcomes of involuntary hospital admission - a review. Acta Psychiatr Scand 2006; 114: 232-41.

58 Katsakou C, Bowers L, Amos T, Morriss R, Rose D, Wykes T, et al. Coercion and treatment satisfaction among involuntary patients. Psychiatr Serv 2010; 61: 286-92.

59 Grinshpoon A, Ponizovsky A. The relationships between need profiles, clinical symptoms, functioning and the well-being of inpatients with severe mental disorders. J Eval Clin Pract 2008; 14: 218-25.

60 Mojtabai R, Fochtmann L, Chang S, Kotov R, Craig T, Bromet E. Unmet need for mental health care in schizophrenia: an overview of literature and new data from a first-admission study. Schizophr Bull 2009; 35: 679-95.

61 Ochoa S, Haro J, Autonell J, Pendas A, Teba F, Marquez M. Met and unme needs of schizophrenia patients in a Spanish sample. Schizophr Bull 2003; 29: 201-10.

62 Priebe S, Reininghaus U, McCabe R, Burns T, Eklund M, Hansson L, et al. Factors influencing subjective quality of life in patients with schizophrenia and other mental disorders: a pooled analysis. Schizophr Res 2010; 121: 251-8.

63 Priebe $S$, McCabe $R$, Junghan $U$, Kallert $T$, Ruggeri M, Slade $M$, et al. Association between symptoms and quality of life in patients with schizophrenia: a pooled analysis of changes over time. Schizophr Res 2011 133: 17-21.

64 Fujii D, Wylie A, Nathan J. Neurocognition and long-term prediction of quality of life in outpatients with severe and persistent mental illness. Schizophr Res 2004; 69: 67-73.

65 Addington J, Addington D. Neurocognitive and social functioning in schizophrenia: a 2.5 year follow-up study. Schizophr Res 2000; 44: 47-56.
66 Brekke J, Kohrt B, Green M. Neuropsychological functioning as a moderator of the relationship between psychosocial functioning and the subjective experience of self and life in schizophrenia. Schizophr Bull 2001; 27 697-708.

67 Green M, Kern R, Heaton R. Longitudinal studies of cognition and functional outcome in schizophrenia: implications for MATRICS. Schizophr Res 2004; 72 : 41-51.

68 Skantze K, Malm U, Dencker SJ, May PR, Corrigan P. Comparison of quality of life with standard of living in schizophrenic out-patients. Br J Psychiatry 1992; 161: 797-801.

69 Smith D, Mar C, Turoff B. The structure of schizophrenic symptoms: a meta-analytic confirmatory factor analysis. Schizophr Res 1998; 31: 57-70.

70 Wegener S, Redoblado-Hodge M, Lucas S, Fitzgerald D, Harris A, Brennan J. Relative contributions of psychiatric symptoms and neuropsychological functioning to quality of life in first-episode psychosis. Aust $N$ Z J Psychiatry 2005; 39: 487-92.

71 Galletly C, Clark C, McFarlane A, Weber D. Relationships between changes in symptom ratings, neurophysiological test performance and quality of life in schizophrenic patients treated with clozapine. Psychiatry Res 1997; 72: 161-6.

72 Ritsner M. Comparison of instruments for measuring the quality of life impairment syndrome in severe mental disorders. In Quality of Life Impairment in Schizophrenia, Mood and Anxiety Disorders: New Perspectives on Research and Treatment (eds M Ritsner, A Awad): 133-42. Springer, 2007.

73 Sota T. Outcome in schizophrenia: are cognitive variables predictors of rehospitalization and quality of life? Diss Abstr Int 2000; 60: 4253.

74 Reininghaus $U$, McCabe R, Burns $T$, Croudace $T$, Priebe $S$. The validity of subjective quality of life measures in psychotic patients with severe psychopathology and cognitive deficits: an item response model analysis. Qual Life Res 2012; 21: 237-46.

75 Cramer J, Rosenheck R, Xu W, Thomas J, Henderson W, Charney D. Quality of life in schizophrenia: a comparison of instruments. Schizophr Bull 2000; 26: 659-66.

76 Lehman A. Measures of quality of life among persons with severe and persistent mental disorders. Soc Psychiatry Psychiatr Epidemiol 1996; 31: 78-88.

77 Van Nieuwenhuizen C, Schene A, Boevink W, Wolf J. Measuring the quality of life of clients with severe mental illness. Psychiatr Rehab J 1997; 20: 33-41.

78 Uher R, Farmer A, Maier W, Rietschel M, Hauser J, Marusic A, et al. Measuring depression: comparison and integration of three scales in the GENDEP study. Psychol Med 2008; 38: 289-300.

79 Cella D, Riley W, Stone A, Rothrock N, Reeve B, Yount S, et al. The PatientReported Outcomes Measurement Information System (PROMIS) developed and tested its first wave of adult self-reported health outcome item banks: 2005-2008. J Clin Epidemiol 2010; 63: 1179-94.

80 Flora D, Curran P. An empirical evaluation of alternative methods of estimation for confirmatory factor analysis with ordinal data. Psychol Methods 2004; 9: 466-91.

81 Embretson S, Reise S. Item Response Theory for Psychologists. Erlbaum, 2000.

82 Brown T. Confirmatory Factor Analysis for Applied Research. Guilford, 2006.

83 Campbell D, Fiske D. Convergent and discriminant validation by the multitrait-multimethod matrix. Psychol Bull 1959; 56: 81-105.

84 Rose M, Bjorner J, Becker J, Fries J, Ware J. Evaluation of a preliminary physical function item bank supported the expected advantages of the Patient-Reported Outcomes Measurement Information System (PROMIS). J Clin Epidemiol 2008; 61: 17-33. 